\section{ScienceDirect}

Materials Today: Proceedings 00 (2014) 000-000 materialstoday:

PROCEEDINGS

www.materialstoday.com/proceedings

5th International Conference on Materials Processing and Characterization

\title{
Image-based software solutions for advanced materials processing and characterization
}

\author{
Yash Agarwal $^{\mathrm{a} *}$, Wojciech Śmigaj ${ }^{\mathrm{b}}$, Philippe G. Young ${ }^{\mathrm{b}}$, Ross Cotton ${ }^{\mathrm{b}}$, Patrick Tompsett ${ }^{\mathrm{b}}$ \\ ${ }^{a}$ Ajmer, Rajasthan, 305 002, India \\ ${ }^{b}$ Simpleware Ltd., Bradninch Hall, Exeter, EX4 3PL, UK
}

\begin{abstract}
New image-based solutions are being developed for advanced materials processing and characterization, which involve working with and creating high-quality models from scan data (such as MRI, CT, micro-CT...). Techniques involving the reconstruction and analysis of 3D materials data can accurately reconstruct internal structures, even to the nano level, and generate high-quality meshes suitable for design and simulation applications. By looking at key techniques and their applications, it is possible to better understand the wider impact and benefits of image-based modelling for industry when working with advanced materials.
\end{abstract}

(C) 2014 The Authors. Elsevier Ltd. All rights reserved.

Selection and peer-review under responsibility of the conference committee members of the 4th International conference on Materials Processing and Characterization.

Keywords: Numerical modelling; materials characterization; image-based modelling; mesh generation; micro-CT

\section{Introduction}

There are many options now available to acquire 3D image data of scanned materials, including computed tomography (CT), micro- and nano-computed tomography (X-ray/nano-CT), and serial sectioning (SEM). By acquiring volume images of complex material structures, it is possible to reconstruct their internal structures. Images can be analysed to obtain qualitative and quantitative information, from visualising cracks and other features, through to acquiring statistics such as porosity and surface area from image data. For advanced and composite materials, image-based techniques are valuable for non-destructive testing, whereby data can be collected without having to damage the original sample or component. When used to complement experimental testing, nondestructive evaluation using 3D image data offers many benefits for fields such as aerospace, automotive and product design.

Novel software techniques have been developed at Simpleware Ltd. (Exeter, UK) for bridging 3D scanning modalities with numerical modelling approaches like Finite Element Analysis (FEA) and Computational Fluid Dynamics (CFD). By working directly with 3D images, it is possible to generate models from very complex geometries, which can then act as the basis for characterisation, and for exporting meshes for simulating different mechanical properties. In particular, these meshes can be used

\footnotetext{
* Corresponding author. Tel.: +919571490995.

E-mail address: y.agarwal@simpleware.com
} 
as part of a process of effective material property calculation based on finite-element simulations. By exploring key techniques, it is possible to understand their value for advanced materials characterisation and processing.

\section{Methodology}

\subsection{Image data acquisition and volume reconstruction}

The first step in creating accurate models from 3D image data involves acquiring a stack of images from scanning modalities such as CT or micro-CT. Images are converted from 2D pixels into 3D pixels (voxels) using image processing software. At this stage, the obtained image data can be processed to segment out regions of interest, including solid, gaseous and fluid phases: see Fig. 1 for an example of material segmentation. Filters can also be applied to best visualise data. Typical software techniques here include thresholding and cropping to isolate selected parts of the image data that are required for the model. This makes it possible, for example, to quickly visualise features of the image data such as cracks and other defects or to identify porous networks.

\subsection{Image analysis and processing}

Comprehensive analysis can be made of material samples by using software tools to extract useful quantitative information from image data. Measurement tools can be used to better understand defects, while statistics can be obtained for properties such as pore sizes, surface areas and volume fractions taken up by individual phases. When combined with more general image processing, it is possible to combine qualitative and quantitative analysis to characterise materials with complex microstructure and multiple phases.

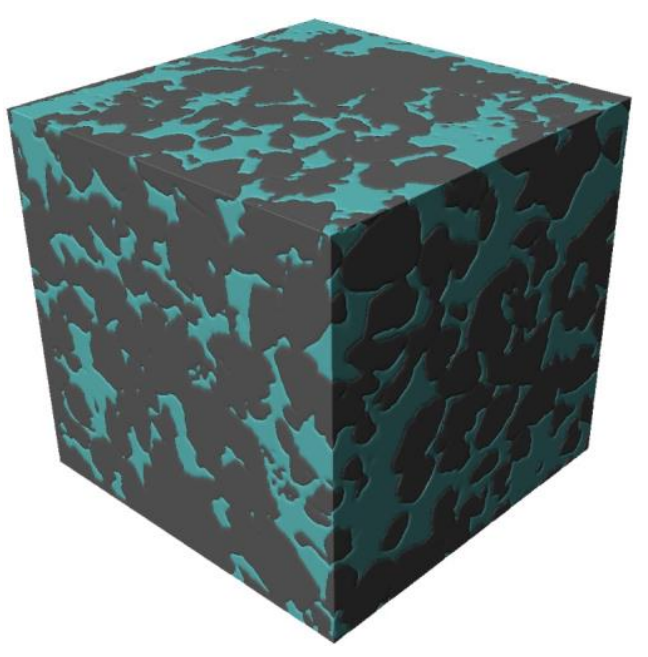

Fig. 1. Segmented micro-CT scan of a dry Berea sandstone sample (data source: [5])

\subsection{Image-based meshing}

3D image data that has been processed and analysed can be rapidly converted into multi-part meshes suitable for comprehensive characterisation of properties, as well as for simulation applications. Image-based meshing approaches hold particular advantages over traditional CAD-based approaches that tend to struggle with complex geometries, requiring extensive user input to make a model ready for simulation. By comparison, image-based methods can generate accurate meshes directly from segmented image data without a loss of volume or topology. The accuracy of image-based meshes is typically only restricted by the quality of original scans, as well as by the software user's ability to segment regions of interest.

Software techniques developed at Simpleware include the use of an 'Enhanced Volumetric Marching Cubes (EVoMaC)' meshing technique by Young et al [1], which adapts the marching cubes algorithm developed by Lorensen and Cline [2] to support multiple segmentation domains. In addition, this technique, which produces very high-quality meshes using a + FE Grid algorithm, can be combined with a multi-part surface remeshing approach (+FE Free). With this approach, voxel-based meshes can be effectively decimated according to the size and complexity of local features. This makes it possible to reduce the mesh size without compromising geometrical accuracy.

The EVoMaC algorithm produces a mixed hexahedral-tetrahedral mesh from the input segmented masks. Voxels lying in mask interiors are converted directly to hexahedral elements, whereas those lying close to mask interfaces are split into tetrahedra to ensure smooth interfaces between model parts in the generated mesh. The surface interpolation quality is enhanced by taking 
advantage of the partial volume information encoded in the input image's greyscale levels to adjust interpolation points. Image smoothing algorithms used in this procedure are volume and topology preserving. If surface remeshing is desired, target minimum and maximum edge lengths are used to decimate the surfaces generated by EVoMaC where possible; the remeshed multi-part surfaces are then filled with tetrahedral elements through an advancing front technique. This approach retains the shape of the original EVoMaC surfaces, maintaining conforming interfaces and shared nodes.

\subsection{Effective material properties calculation}

Image-based meshing techniques can also be used for calculating effective material properties from material samples. The aim is to approximate a complex heterogeneous material, for example a multi-phase composite, with a homogeneous material with 'effective' properties chosen so that its response to external loading resembles as closely as possible that of the original material. By using this approach, it is possible to simplify the often difficult process of working with materials image data; the obtained effective material properties enable a link to be created between micro- and macro-scale modelling.

Effective material properties were traditionally calculated by approximating them by one of a small number of geometries whose effective properties were known analytically, for example as ensembles of spherical particles. Alternatively, upper and lower bounds for the effective properties can be derived from the properties and volume fractions (or higher-order correlation functions) of individual constituent phases [3]. Recent increases in available computing power have made it possible, however, to calculate the effective properties by numerically solving a family of boundary value problems imposed on a sample of the chosen material. By taking this approach, the often complex geometry of composites can be fully taken into account, while the problem of looseness of bounds obtained for composites with highly contrasting phases can be solved.

As the geometry of scanned samples of composite materials is given in the form of segmented raster images, numerical homogenisation is typically carried out by solving the necessary boundary value problems with methods based on Cartesian grids. With this approach, no separate meshing step is required, with Cartesian grid methods also being suitable for GPU acceleration and matrix-free algorithms. However, solutions of Cartesian-grid discretisations of boundary value problems involving linear elliptic partial differential equations with discontinuous coefficients converge only linearly with image resolution. Convergence problems are exacerbated for physical properties sensitive to surface area, as the surface area of the voxel mesh approximating a smooth body may not converge to the exact surface area of the latter.

Homogenisation techniques developed by Simpleware for their Physics Modules use a built-in finite element solver to calculate the response of a cuboidal sample of a material to a sequence of boundary conditions. The very robust image-based meshing techniques described above produce body-conforming meshes, which improves the convergence rate of the effective properties: accurate results can be obtained using a smaller number of unknowns than with Cartesian grids. The three available Physics Modules, +SOLID, +LAPLACE and +FLOW, enable calculation effective elastostatic moduli, thermal and electrical conductivity, electrical permittivity, molecular diffusivity and absolute permeability.

\section{Validation of methods}

The quality of the meshing approach and the calculation of effective material properties has been validated [4] using freely available rock physics data from ETH, Zurich, as well as results published from this data in two installments by Andra et al. [5; 6]. Simpleware software was used, in this case, to import, process and segment the image data, generate meshes and calculate effective properties. The data was acquired from micro-CT scans of a dry Berea sandstone sample, encoded on a grid of $1024 \mathrm{x} 1024 \mathrm{x} 1024$ cubic voxels with side length $0.74 \mu \mathrm{m}$. Simpleware ScanIP was used to reconstruct the 16-bit scan data, imported as a stack of 2D TIF images. The effect of reconstruction artefacts on the quality of the images was reduced by filtering and segmenting the images; this included dealing with streaks and brightness non-uniformity, and the removal of a layer of 150 voxels on each of the $\mathrm{x}$ and $\mathrm{y}$ faces, giving a new sample size of 724 x 724 x 1024 voxels.

Different image processing tools were used to segment the image data. A median filter was used to reduce noise, as shown in Fig. 2, following methods carried out in work by Andra et al. [5; 6]. Segmentation identified three phases in the Berea sandstone sample: quartz, mineral inclusions, and the air occupying the pores. The threshold greyscale range was chosen so that the effect of the phase-contrast fringe artefact, occurring at the grain-air interfaces, did not skew porosity and the resulting effective properties of the sandstone. An island removal tool was applied to reassign remaining unconnected small-scale features in the segmented phases to the relevant phase. An additional proprietary smart mask smoothing algorithm was applied to the masks; this technique maintains the volume and topology of the phases. 

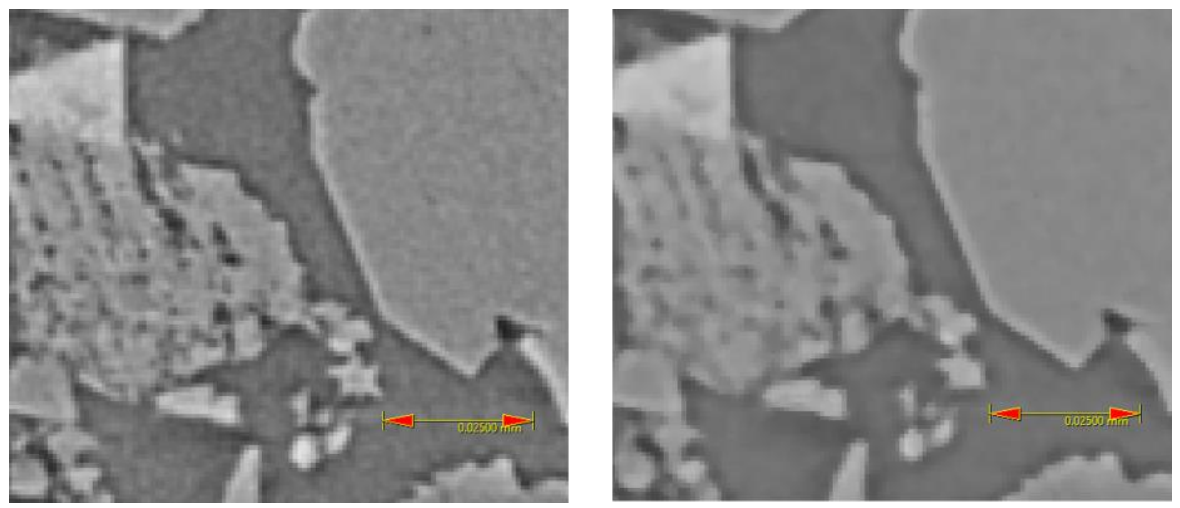

Fig. 2. Berea sandstone before (left) and after applying the median filter (right).

The model-based volume fraction statistic tool was used to determine the porosity of the rock sample. In contrast to voxel based statistics, which are calculated directly from the voxels included in the segmented phases, model-based statistics carry out a quick 3D model rendering in the background to include partial volumes generated during filtering and segmentation. Model based statistics are therefore generated from a physically accurate model with a smooth outer surface. We measured a porosity of 0.181 for the complete $724 \times 724 \times 1024$ voxel rock sample; the sub-regions of interest used for the homogenisation were measured to have porosities of 0.172 and 0.173 . The values calculated by ScanIP for the segmentation method used in the validation show good agreement with the benchmark results from [5], which put the porosity of the sample in question at 0.184 .

A mesh density study on a $175 \times 175 \times 175$ voxel sample of the sandstone was carried out, which demonstrated the high degree of control over mesh element sizes provided by Simpleware software, while also preserving accurate representations of the sample in terms of the porosity. The model-based volume fraction tool was used to calculate a benchmark porosity for the 175 voxel region of interest, resulting in a porosity value of 0.1687 . See Table 1 for a summary of the general statistics associated with the generated meshes, including the porosity and its deviation from the benchmark value.

Table 1. Mesh statistics

\begin{tabular}{llllll}
\hline Mesh setting & $\begin{array}{l}\text { No. of } \\
\text { elements }\end{array}$ & No. of nodes & $\begin{array}{l}\text { Porosity } \\
(\%)\end{array}$ & $\begin{array}{l}\text { Porosity error } \\
(\%)\end{array}$ & $\begin{array}{l}\text { Meshing time } \\
(\mathrm{s})\end{array}$ \\
\hline +FE Grid & $11.636 \mathrm{~m}$ & $2,233 \mathrm{k}$ & 16.83 & 0.23 & 142 \\
+FE Free -10 & $2.844 \mathrm{~m}$ & $551 \mathrm{k}$ & 16.82 & 0.29 & 939 \\
+FE Free -25 & $1.093 \mathrm{~m}$ & $203 \mathrm{k}$ & 16.82 & 0.26 & 399 \\
+FE Free -50 & $816 \mathrm{k}$ & $146 \mathrm{k}$ & 16.82 & 0.28 & 315 \\
+FE Free Custom & $503 \mathrm{k}$ & $91 \mathrm{k}$ & 16.81 & 0.35 & 250 \\
\hline
\end{tabular}

Even after a $23 x$ reduction in the number of elements used in the mesh, the porosity error only increased by as little as $0.12 \%$ when comparing the $+\mathrm{FE}$ Grid and + FE Free custom meshes. A range of mesh quality metrics were also recorded based on different mesh settings, including the in-out aspect ratio, Jacobian and edge length aspect ratio, to demonstrate the overall quality of the meshes changes little as the number of elements are reduced. All of the mesh density studies were conducted using a standard workstation with $32 \mathrm{~GB}$ of RAM and an Intel Core i7 processor.

To calculate effective material properties relevant to elasto-statics, viscous fluid flow and current flow, two regions of interest were used to reduce simulation memory and processing time when working with the Berea sandstone. The $724 \times 724 \times 1024$ voxel sample was cropped to X: 195-595, Y: 195-595, Z: 100-500 (a) and X: 195-595, Y: 195-595, Z: $500-900$ (b); this resulted in $400 \times 400 \times 400$ voxel cubes positioned in the central upper and lower sections of the sample.

The quartz phase of the segmentation was modelled for the study of elasto-static properties. Simulations were performed using kinematic and mixed uniform boundary conditions. Young's modulus and Poisson's ratio of quartz were taken from the literature [5]. Table 2 compares the isotropic effective material properties obtained using the Simpleware module +SOLID to the finite element method (FEM) results from [6]. 
Table 2. Effective elasto-static properties

\begin{tabular}{rrll}
\hline ROI & Boundary condition & $\begin{array}{l}\text { Effective Young's } \\
\text { modulus (GPa) }\end{array}$ & $\begin{array}{l}\text { Effective Poisson's } \\
\text { ratio }\end{array}$ \\
\hline z100-500 & Kinematic uniform & 49.98 & 0.0956 \\
& Mixed uniform & 47.87 & 0.1072 \\
\hline z500-900 & Kinematic uniform & 51.03 & 0.0959 \\
& Mixed uniform & 49.11 & 1.1058 \\
\hline Mean & 49.50 & 0.1011 \\
\hline Ref. 6 (FEM simulations) & 46.01 & 0.1166 \\
\hline
\end{tabular}

Four simulations produced mean values of $49.50 \mathrm{GPa}$ and 0.1011 for the effective Young's modulus and Poisson's ratio. The values compare well with the values of $46.01 \mathrm{GPa}$ and 0.1166 obtained in [6].

The fluid phase surrounding the quartz was then modelled for the viscous fluid flow simulations to calculate the absolute permeability of the sandstone in Simpleware module +FLOW, using the following boundary conditions for each of the ROIs: linear pressure variation on side walls, impermeable side walls and solid side walls. The isotropic approximation to the absolute permeability are given in Table 3, alongside the Lattice Boltzmann method (LBM) results from [6].

Table 3. Absolute permeability

\begin{tabular}{rrl}
\hline ROI & Boundary condition & $\begin{array}{l}\text { Absolute permeability } \\
(\mathrm{mD})\end{array}$ \\
\hline z100-500 & Linear pressure variation & 124.40 \\
& Impermeable & 113.10 \\
& Solid & 113.10 \\
\hline z500-900 & Linear pressure variation & 77.26 \\
& Impermeable & 67.14 \\
Solid & 66.56 \\
\hline Mean & 93.59 \\
\hline Ref. 6 (LBM simulations) & 113.00 \\
\hline
\end{tabular}

The fluid flow results show a large spread of values between the physical properties between the two ROIs. The z100-500 ROI gives a mean absolute permeability that compares well with the value from [6], but the mean absolute permeability for the z500-900 ROI shows a significant difference, highlighting the effect of the choice of ROI.

In the characterisation of electric transport properties of the two ROIs, the intergrain space was assumed to be filled with water. Calculations were performed with the Simpleware module +LAPLACE using the Dirichlet and mixed uniform boundary conditions on the surface of each of the ROIs. The results are expressed in terms of the resistivity formation factor, defined as the ratio of the electrical conductivity of the conducting phase to the effective conductivity of the composite. This quantity does not depend on the conductivity of the conducting phase, but only on its geometry. In Table 4 below the values obtained with +LAPLACE are juxtaposed with the results of FEM simulations from [6].

Table 4. Effective electrical properties

\begin{tabular}{rrl}
\hline ROI & Boundary condition & Resistivity formation factor \\
\hline z100-500 & Dirichlet uniform & 20.67 \\
& Mixed uniform & 23.52 \\
\hline z500-900 & Dirichlet uniform & 21.06 \\
& Mixed uniform & 23.61 \\
\hline Mean & 22.22 \\
\hline & Ref. 6 (FEM simulations) & 21.10 \\
\hline
\end{tabular}

Again, the mean of the simulation results, 22.22, compares well to the value of 21.10 reported by [6]. The results are therefore in general agreement with the reference paper. It should be noted that the Physics Modules calculate not only the isotropic 
approximation to the effective material properties, but the full effective tensor, which is needed for the description of the behaviour of anisotropic materials. The fields obtained by solving the boundary value problems used to calculate the effective properties can also be visualised in Simpleware software and exported in numerical or image form. An example is shown in Fig. 3.

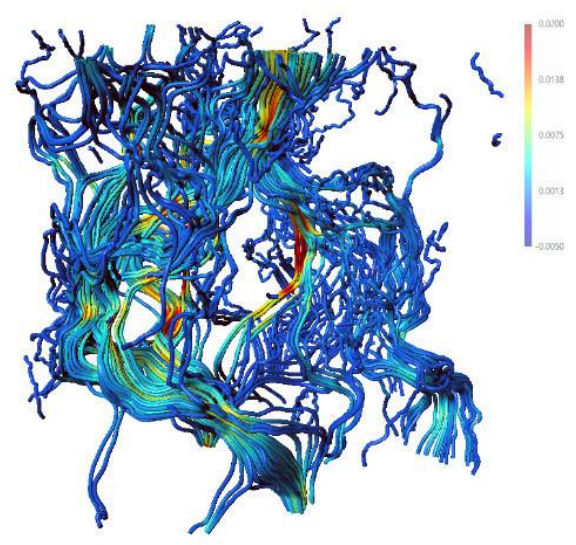

Fig. 3. Fluid velocity streamlines obtained in a fluid flow simulation

\section{Conclusion}

Image-based modelling of materials samples and calculation of effective material properties using homogenisation methods demonstrates great potential for characterisation workflows. Being able to non-destructively analyse materials and gain quantitative information on their properties means that detailed characterisation can be carried out. The examples given in this paper from digital rock physics show the strength of the approach for working with complex materials image data. The ability to integrate different stages of this workflow into a single software environment is particularly beneficial for speeding up the process of going from a 3D image through to models ready for simulation; these workflows have many benefits for materials scientists, analysts and technicians working in fields such as composites, oil and gas and any other application involving 3D imaging of material samples.

\section{References}

[1] P.G. Young, T.B.H. Beresford-West, S.R.L. Coward, B. Notarberardino, A. Walker, A. Abdul-Aziz, Phil. Trans. Royal Soc. A. 366 (2008) 3155-3173.

[2] W.E. Lorensen, H.E. Cline, Computer. Graph. 21 (1987) 163-69.

[3] Z. Hashin, J. Appl. Mech. 50 (1983) 481-505.

[4] R. Cotton, P. Tompsett, W. Śmigaj, K. Genc, P.G. Young, NAFEMS World Cong., 21-24 June, San Diego, CA. (2015).

[5] H. Andrä, N. Combaret, J. Dvorkin, E. Glatt, J. Han, M. Kabel, Y. Keehm, F. Krzikalla, M. Lee, C. Madonna, M. Marsh, T. Mukerji, E. Saenger, R. Sain, N. Saxena, S. Ricker, A. Wiegmann, X. Zhan, Computers \& Geosci. 50 (2013a) 25-32.

[6] H. Andrä, N. Combaret, J. Dvorkin, E. Glatt, J. Han, M. Kabel, Y. Keehm, F. Krzikalla, M. Lee, C. Madonna, M. Marsh, T. Mukerji, E. Saenger, R. Sain, N. Saxena, S. Ricker, A. Wiegmann, X. Zhan, Computers \& Geosci. 50 (2013b) 33-43. 